\title{
Linking local retention, self-recruitment, and persistence in marine metapopulations
}

\author{
Christophe Lett, ${ }^{1,7}$ Tri Nguyen-Huu, ${ }^{2}$ Marion Cuif, ${ }^{1,3,4}$ Pablo Saenz-Agudelo, ${ }^{5,6}$ and David M. Kaplan ${ }^{3,8}$ \\ ${ }^{1}$ UMI IRD 209 UPMC UMMISCO, Centre de Recherche Halieutique Méditerranéenne et Tropicale, BP 171, 34203 Sète Cedex, France \\ ${ }^{2}$ UMI IRD 209 UPMC UMMISCO, IXXI, 7 Rue du Vercors, 69007 Lyon, France \\ ${ }^{3}$ UMR EME 212, IRD, Centre de Recherche Halieutique Méditerranéenne et Tropicale, BP 171, 34203 Sète Cedex, France \\ ${ }^{4}$ UR COREUS, IRD, BP A5 98848 Nouméa Cedex, New Caledonia \\ ${ }^{5}$ Red Sea Research Center, King Abdullah University of Science and Technology, Thuwal, Jeddah 23955 Kingdom of Saudi Arabia \\ ${ }^{6}$ Instituto de Ciencias Ambientales y Evolutivas. Universidad Austral de Chile, Valdivia, Chile
}

\begin{abstract}
Three indices of larval retention have been used in the literature to assess the tendency for self-maintenance of local marine populations: local retention (LR), selfrecruitment (SR), and relative local retention (RLR). Only one of these, LR, defined as the ratio of locally produced settlement to local egg production, has a clear relationship to selfpersistence of individual sites. However, SR, the ratio of locally produced settlement to settlement of all origins at a site, is generally easier to measure experimentally. We use theoretical, simulation, and empirical approaches to bridge the gap between these different indices, and demonstrate that there is a proportional relationship between SR and LR for metapopulations close to a stable state and with lifetime egg production (LEP) approximately uniform over space. Similarly, for systems where larval mortality rates are a relatively uniform function of release site, RLR (defined as the ratio of locally produced settlement to all settlement of local origin) and LR will also be proportional. Therefore, SR and RLR provide information on relative rates of LR for systems satisfying these conditions. Furthermore, the ratio between LR and SR can be used to evaluate global persistence of metapopulations, and therefore provides valuable information not necessarily available if only LR is considered.
\end{abstract}

Key words: connectivity; larval dispersal; lifetime egg production; local retention; marine reserve; metapopulation; network persistence; population persistence; self-persistence; self-recruitment.

\section{INTRODUCTION}

Based on modeling work and a limited number of empirical results, Cowen et al. (2000) stated 15 years ago that marine populations may not be as open as previously thought. Since then, this statement has been supported by several field studies assessing the ratio of locally produced settlement to settlement of all origins arriving at a given settlement site, generally referred to by the term self-recruitment (SR; Botsford et al. 2009). Unexpectedly high values of SR have been regularly reported (e.g., Jones et al. 1999, 2005, Almany et al. 2007, Hamilton et al. 2008, Planes et al. 2009, Carson et al. 2010, Saenz-Agudelo et al. 2011, 2012, Hogan et al. 2012), suggesting that marine systems are less open than historically assumed (SR is equal to one minus "openness" as defined by Hixon et al. [2002] and Pinsky et al. [2012]). However, Burgess et al. (2014) recently

Manuscript received 7 July 2014; revised 13 January 2015; accepted 5 February 2015. Corresponding Editor: B. D. Inouye.

7 E-mail: christophe.lett@ird.fr

${ }^{8}$ Present address: Virginia Institute of Marine Science, College of William \& Mary, P.O. Box 1346, Gloucester Point, Virginia 23062 USA. demonstrated that these high empirical SR estimates do not assure self-persistence of individual recruitment sites (i.e., that sites are "closed" in the sense of being selfsustaining). Rather, the ratio of locally produced settlement to total local larval release, referred to as local retention (LR; Botsford et al. 2009), is necessary to test for self-persistence of individual sites. But LR is difficult to assess experimentally because it depends on the number of eggs produced at each site, which is often poorly known. Furthermore, its interpretation in terms of persistence requires a measure of the lifetime egg production (LEP) of an average recruit to the adult population (Burgess et al. 2014), which is not always available. Finally, when LR is insufficient to ensure selfpersistence of individual recruitment sites, global network persistence of the marine metapopulation depends on the full connectivity matrix describing probability of larval exchanges between all pairs of sites (Burgess et al. 2014), which is even more complex to measure.

These considerations call into question the value of empirical observations of SR for assessing persistence of populations, e.g., in the context of marine spatial management. Recently, Hogan et al. (2012) proposed to assess local retention using another measure: the ratio 
of locally produced settlement to the total number of settlers locally produced, here referred to by the term relative local retention (RLR). Hogan et al. (2012) found that RLR values were similar in magnitude to SR values measured at the same sites, and therefore concluded that "self-recruitment might be a good estimator of [relative] local retention." This conclusion is interesting because, if it is widely applicable and RLR values are informative of LR values, then SR measurements provide valuable indices of self-persistence that are relatively easy to measure empirically. However, the robustness of this empirically observed relationship has yet to be tested in other systems and its consequences for persistence in marine metapopulations are uncertain, due to the lack of a clear theoretical understanding of the relationships between the indices of retention.

The objective of the present study is to identify theoretical and empirical links between SR, LR, and RLR, as well as assess their utility for understanding population persistence. We begin by mathematically defining the three measures and exploring the theoretical relationships between them in the context of a marine metapopulation connected by larval dispersal. These relationships are then tested using two different approaches: simulated marine metapopulations with arbitrary patterns of connectivity, and empirical observations of connectivity. As connectivity is frequently measured some time after recruitment into the juvenile population has occurred (Pineda et al. 2007), we also examine theoretically and via simulations the consequences of post-settlement density-dependent recruitment for retention indices and persistence assessments. This study therefore bridges the gap between theoretical and empirical assessments of these variables, and provides information directly relevant to the use of these retention indicators for management of marine ecosystems.

\section{Theoretical Development}

\section{Definition of retention indicators}

As the terminology used for the retention indicators described in Introduction varied in the literature, it is useful to begin with some mathematical definitions of the three indicators. Let us consider a marine metapopulation with planktonic early life stages (eggs and larvae) and sedentary juveniles and adults living in a patchy environment. We suppose that we have a set of $n$ patches for egg release and larval recruitment. For $i, j \in\{1,2$, $\ldots, n\}$, if we define $\mathbf{N}_{t}=\left(N_{i, t}\right)$ to be the vector containing the number of eggs produced at each patch $i$ at time $t$, and $\mathbf{C}=\left(c_{j i}\right)$ to be the connectivity matrix describing the proportion of eggs released at patch $i$ that recruit into the juvenile population at patch $j$ (Pineda et al. 2007), then local retention on patch $i, \mathrm{LR}_{i}$, is the ratio of larval recruitment in this patch $\left(c_{i i} N_{i, t}\right)$ to local egg production $\left(N_{i, t}\right)$

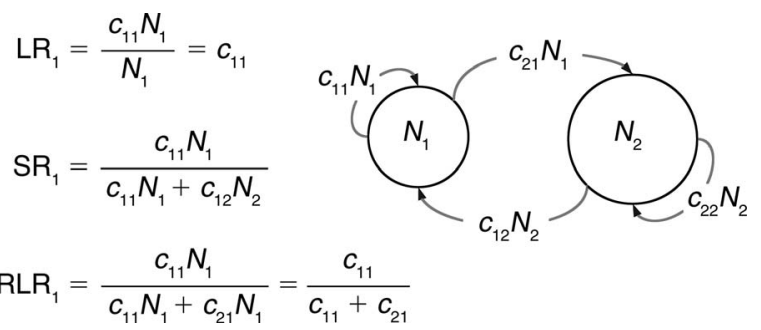

FIG. 1. Local retention (LR), self-recruitment (SR), and relative local retention (RLR) as defined for patch 1 of a schematic two-patch metapopulation. $N_{i}$ is the number of eggs released at patch $i_{j}$ and $c_{i j}$ is the proportion of these eggs that recruit into the juvenile population at patch $j$.

$$
\mathrm{LR}_{i}=\frac{c_{i i} N_{i, t}}{N_{i, t}}=c_{i i}
$$

As noted by Botsford et al. (2009), LR is equal to the diagonal elements of the connectivity matrix. It does not depend on egg production $\left(\mathbf{N}_{t}\right)$, meaning it is independent of temporal changes in adult population size.

For relative local retention on patch $i, \mathrm{RLR}_{i}$, the denominator of the ratio is replaced by the total number of larvae of local origin that recruit into all habitat patches

$$
\operatorname{RLR}_{i}=\frac{c_{i i} N_{i, t}}{\sum_{j=1}^{n} c_{j i} N_{i, t}}=\frac{1}{\sum_{j=1}^{n} c_{j i}} \operatorname{LR}_{i} .
$$

RLR does not depend on egg production, but it does depend on the connectivity between the focal patch and the ensemble of patches in the system.

Self-recruitment on patch $i$ at time $t, \mathrm{SR}_{i, t}$, is the ratio of locally produced recruits to all local recruitment

$$
\mathrm{SR}_{i, t}=\frac{c_{i i} N_{i, t}}{\sum_{j=1}^{n} c_{i j} N_{j, t}}=\frac{1}{\sum_{j=1}^{n} c_{i j} N_{j, t} / N_{i, t}} \mathrm{LR}_{i} .
$$

Unlike LR and RLR, SR depends on egg production in the different habitat patches (relative to that of the focal patch) and therefore on temporal changes in population size. SR also depends on connectivity between every patch and the focal patch.

Fig. 1 shows LR, SR, and RLR as defined for patch 1 of a schematic two-patch metapopulation.

\section{Links among retention indicators and their relevance for persistence}

In order to understand the links between these retention indicators, it is necessary to place them in the context of a full life-cycle metapopulation model. In addition to the vector of egg production levels in each patch, $\mathbf{N}_{t}$, and the connectivity matrix, $\mathbf{C}$, we assume that a recruit to patch $j$ has an average lifetime egg production (LEP) of $e_{j}$. 
Assuming non-overlapping time-discrete generations, the metapopulation dynamics are described by the following system for each generation $t$ :

$$
\begin{gathered}
\mathbf{N}_{t+1}=\mathbf{M N}_{t} \\
\text { with } \mathbf{N}_{t}=\left(\begin{array}{c}
N_{1, t} \\
N_{2, t} \\
\vdots \\
N_{n, t}
\end{array}\right), \mathbf{M}=\left(\begin{array}{cccc}
m_{11} & m_{12} & \ldots & m_{1 n} \\
m_{21} & m_{22} & \ldots & m_{2 n} \\
\vdots & & \ddots & \vdots \\
m_{n 1} & \ldots & \ldots & m_{n n}
\end{array}\right) \text {, } \\
\text { and } m_{j i}=e_{j} c_{j i}
\end{gathered}
$$

where $\mathbf{M}$ is a matrix that has elements $\left(m_{j i}\right)$ that are the product of egg production $\left(e_{j}\right)$ and connectivity $\left(c_{j i}\right)$. This model also applies asymptotically (i.e., after many generations) to age-structured populations with overlapping generations (Kaplan et al. 2006). Initially, we will assume that $\mathbf{C}$ is density independent, but this assumption will be relaxed later on.

First note that if one ignores larval dispersal between different patches by setting off-diagonal elements of the connectivity matrix to zero, then the dynamics reduce to $N_{i, t+1}=e_{i} c_{i i} N_{i, t}$. Therefore, egg production will be stable or increasing in a patch if $e_{i} c_{i i} \geq 1$, or in other terms, LEP $\times$ LR $\geq 1$. This is the self-persistence condition described by Burgess et al. (2014).

As the definitions of SR and RLR involve connectivity between distinct patches, their relationships to LR and population persistence can only be understood by examining the dynamics of the full system. At an arbitrary time, little link is evident between SR and LR because SR depends on the population state vector $\mathbf{N}_{t}$. However, asymptotically, our population model reduces to $\mathbf{N}_{t+\mathbf{1}}=\lambda_{\mathbf{M}} \mathbf{N}_{t}$ with $\lambda_{\mathbf{M}}$ the largest eigenvalue of $\mathbf{M}$. If LEP is uniform over space, then $\lambda_{\mathbf{M}}=e \lambda_{\mathbf{C}}$ where $\lambda_{\mathbf{C}}$ is the largest eigenvalue of $\mathbf{C}$ and $e>0$ is the uniform LEP value. In this case, we asymptotically obtain $\mathbf{N}_{t+1}=e \mathbf{C N}_{t}$ $=e \lambda_{\mathbf{C}} \mathbf{N}_{t}$, i.e., $\sum_{j=1}^{n} c_{i j} N_{j, t}=\lambda_{\mathbf{C}} N_{i, t}$ for any patch $i$. Substituting in Eq. 3, one finds that $\mathrm{LR}=\lambda_{\mathbf{C}} \mathrm{SR}$, i.e., $\mathrm{LR}$ is proportional to $\mathrm{SR}$ on all patches with $\lambda_{\mathbf{C}}$ playing the role of constant of proportionality. When LEP varies over space with standard deviation $\sigma$, the relationship still holds approximately on each patch with $\mathrm{LR}=\lambda_{\mathbf{C}} \mathrm{SR}$ $+\mathrm{O}(\sigma)$, where $\mathrm{O}$ (Landau's symbol) refers to big $\mathrm{O}$ notation, i.e. the error made when using the approximation is proportional to $\sigma$ (see mathematical proof in Appendices A and B).

A direct consequence of this relationship is that the condition for global persistence of the metapopulation, $\lambda_{\mathbf{M}} \geq 1$ (by the Perron-Frobenius theorem), becomes LEP $\times$ LR/SR $\geq 1$ equally on all patches asymptotically when LEP is uniform over space. If LEP varies over space, then the conditions $\overline{\mathrm{LEP}} \times \mathrm{LR} / \mathrm{SR} \geq 1$ assessed on multiple patches, where $\overline{\text { LEP }}$ is the spatial mean of LEP, are valid approximate conditions for global persistence of the metapopulation as long as $\sigma$ is small (see Appendix A).

RLR has a similar approximately proportional relationship to $\mathrm{LR}$, with variability in larval survival between release sites (i.e., the sums of the columns of $\mathbf{C}=\sum_{j=1}^{n} c_{j i}$ ) playing a similar role to that played by spatial variability in LEP for the relationship between LR and SR.

\section{Effects of collecting settlers vs. recruits}

Until now, we have made no separation between settlers, i.e., larvae immediately after the planktonic dispersal phase, and recruits, i.e., larvae that have survived potentially density-dependent early post-settlement mortality and are ready to enter the juvenile population. Whereas numerical larval dispersal studies generally only attempt to simulate the prior (e.g., Cuif et al. 2014), most empirical studies collect individuals sometime after settlement occurs (Pineda et al. 2007).

To understand how this relates to retention indices and persistence estimates, it is necessary to separate the connectivity matrix $\mathbf{C}$ into two terms: a term accounting for physical transport and pre-settlement larval mortality, and a term for post-settlement mortality up until recruitment. If we define $\mathbf{D}=\left(d_{j i}\right)$ to be the dispersal matrix representing the proportions of eggs released at some patch $i$ that survive to settlement at some patch $j$, and $f_{j}$ to be the (potentially density-dependent) probability that a settler in patch $j$ survives to recruitment, then $c_{j i}=f_{j} d_{j i}$ and

$$
\begin{aligned}
\mathrm{LR}_{i} & =f_{i} d_{i i} \\
\mathrm{RLR}_{i} & =\frac{f_{i} d_{i i}}{\sum_{j=1}^{n} f_{j} d_{j i}} \\
\mathrm{SR}_{i, t} & =\frac{f_{i} d_{i i} N_{i, t}}{\sum_{j=1}^{n} f_{i} d_{i j} N_{j, t}}=\frac{d_{i i} N_{i, t}}{\sum_{j=1}^{n} d_{i j} N_{j, t}} .
\end{aligned}
$$

Here, it is implicitly assumed that post-settlement survival does not depend on the origin of the larvae.

Assuming now that individuals are collected as settlers, instead of recruits, leads to omitting all $f$ values in the above equations. This shows that (1) LR decreases if recruits instead of settlers are collected due to the larval mortality included in $f$, (2) RLR will generally differ if recruits vs. settlers are collected (unless all $f$ values are identical, i.e., all the different sites have equal post-settlement survivorship), and (3) SR is the same for individuals collected as settlers or recruits (unless settlers of different origins have different post-settlement survivorship; Vigliola and Meekan 2002, Hamilton et al. 2008). These observations have consequences for density-dependent recruitment. If an increase in the number of settlers leads to a decrease in the $f$ values due 
to negative density dependence, then LR based on the number of recruits will decrease at high recruitment levels. Changes in RLR due to density dependence are more difficult to predict, as they depend on the relative rates of post-settlement mortality in habitat patches. Though SR does not depend directly on the $f$ values, it depends indirectly on them via the egg production levels in the habitat patches, and, therefore, it may vary as a function of density dependence.

For persistence assessments, it is necessary that LEP is calculated from the point in the life cycle that individuals are collected for assessing retention (i.e., at settlement or recruitment), so that LEP accounts for survival from this point to death while retention indicators account for survival from egg to this point (Burgess et al. 2014).

\section{Application of Theory}

The robustness of the theory was tested in two ways: using empirical observations and using simulated random patterns of connectivity.

\section{Empirical data}

To our knowledge, Carson et al. (2010), SaenzAgudelo et al. (2011, 2012), and Hogan et al. (2012) are the only experimental studies to have assessed a connectivity matrix, i.e., exchanges of individuals between multiple, spatially distinct sites, over several years, providing valuable data sets to test a robust empirical link between SR, LR, and RLR in marine metapopulations. However, only Saenz-Agudelo et al.'s $(2011,2012)$ empirical connectivity data is available in an unabridged, multiyear format, and, therefore, these data are examined here. Saenz-Agudelo et al. (2011, 2012) studied connectivity among eight spatially distinct subpopulations of the panda clownfish Amphiprion polymnus in Papua New Guinea (see Plate 1) over three years (2008-2010). From Saenz-Agudelo et al.'s (2011, 2012) data set, we added the summer and winter connectivity matrices of each year and then calculated for each year and sampling site (1) SR, as they did, dividing the number of locally produced recruits (juveniles in Saenz-Agudelo et al.'s [2011, 2012] studies) by the number of recruits of all origins (i.e., diagonal element of a matrix divided by the sum of elements in the corresponding row), (2) RLR, dividing the number of locally produced recruits by the total number of recruits locally produced (i.e., diagonal element of a matrix divided by the sum of elements in the corresponding column), and (3) LR, dividing the number of locally produced recruits by the number of adults (referred to as breeders in Saenz-Agudelo et al. 2011). For calculating LR, we assumed that the number of adults is proportional to the number of eggs produced.

LR, SR, and RLR estimates were compared by first removing from the data set the sites sampled by SaenzAgudelo et al. $(2011,2012)$ for which $S R=L R=R L R=0$. We then analyzed the relationship between LR and SR, LR and RLR, and SR and RLR over all sites using a linear model for each of the three different years $(2008, n=7$; $2009, n=7 ; 2010, n=5)$. In all cases, the intercept of the best-fitted linear model was not significantly different from $0(P>0.05)$. Therefore, we finally analyzed all the relationships with a linear model forced through $(0,0)$. We used the same approach for a limited number of simulations. All statistics were computed using $\mathrm{R}$ version 2.15.0 (R Development Core Team 2012).

\section{Simulations}

Simulations were used to investigate quantitatively the effect of variable LEP values among patches on the relationships between LR, SR, and RLR. Simulated connectivity matrices $\mathbf{C}$ with elements chosen randomly from a uniform distribution between 0 and 1 were generated for a system with eight habitat patches. Values of LEP, $e_{j}$, were chosen randomly from a gamma distribution with mean 1 and different values of standard deviation $\sigma$ covering the interval $[0,1]$. The dominant eigenvalue $\lambda_{\mathbf{M}}$ of the matrix $\mathbf{M}$ was then calculated and $\mathbf{M}$ was multiplied by $1.001 / \lambda_{\mathbf{M}}$ to avoid the population decreasing or growing too fast. The dynamical system in the absence of density-dependent recruitment (Eq. 4) was then run through time for 10000 time steps, ensuring the convergence to the asymptotic stable spatial distribution. At the end of each simulation, the values of LR, SR, and RLR were calculated along the eight patches and the correlation coefficient between these indices assessed. Linear models were also calculated for a limited number of simulation runs using the same procedure as for empirical data.

\section{Density dependence}

Density-dependent recruitment was integrated into our model formulation by making the proportion of settlers surviving as recruits, $f_{j}$, a function of the settlement rate. To evaluate the impact of negative density dependence on retention indices, the dispersal matrix was configured as in Simulations, except that LEP was assumed uniform over space in order to isolate the effect of density dependence. A single Beverton-Holt settler-recruit relationship was used for calculating the $f_{j}$ at all sites. The relationship was configured so that the entire system collapses when LEP is less than $35 \%$ of the maximum value (Kaplan et al. 2009). The uniform value of LEP was varied, with larger values leading to increased negative density dependence. Note that the use of a single settler-recruit relationship does not imply that the $f_{j}$ are uniform over space, as the value of $f_{j}$ depends on the settler-recruit relationship and the settlement rate at a given site, which will vary over space due to differences in connectivity.

\section{RESUlTS Empirical data}

The values of SR, LR, and RLR calculated from Saenz-Agudelo et al.'s $(2011,2012)$ data are reported in Appendix C. A strong linear relationship is observed 

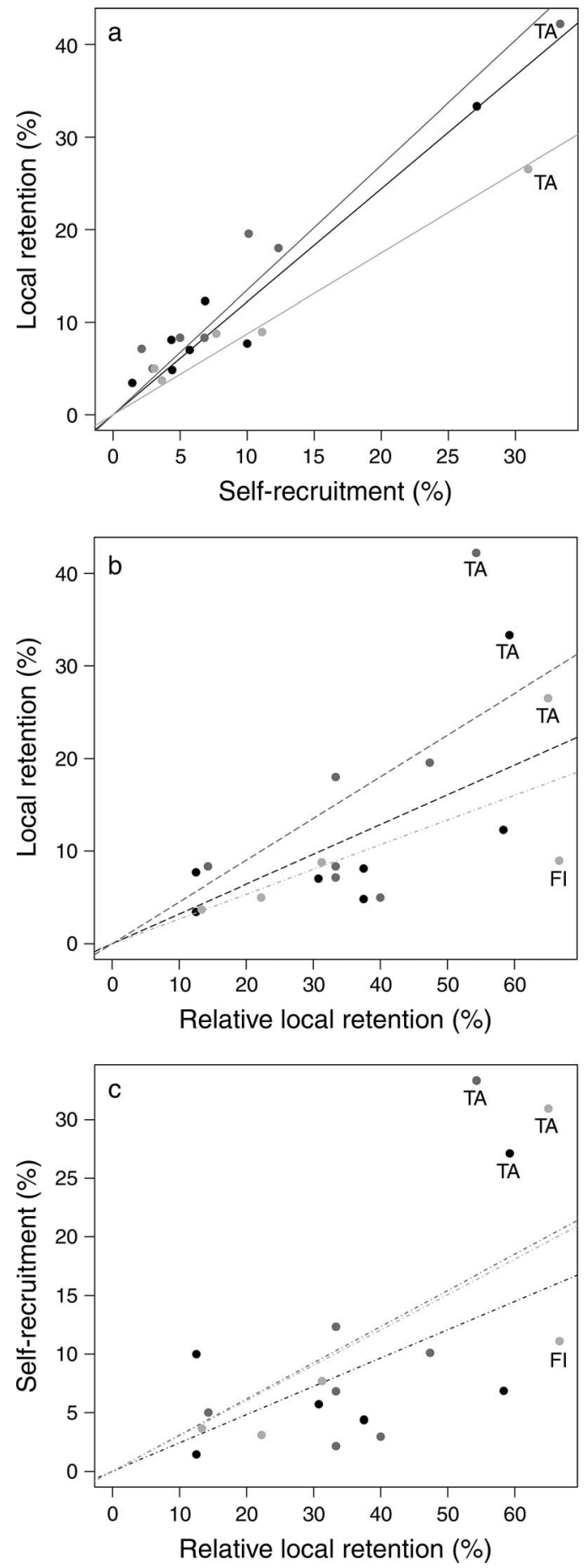

FIG. 2. Plots of Saenz-Agudelo et al.'s $(2011,2012)$ data (Appendix C) for years 2008 (black), 2009 (dark gray), and 2010 (light gray): (a) local retention (LR) vs. self-recruitment (SR), (b) LR vs. relative local retention (RLR), (c) SR vs. RLR. Best-fitted linear models with a slope significantly different from $0(P<0.001$, solid line; $P<0.01$, dashed line; $P<0.05$, dot-dashed line) are shown. Data from the TA and FI sites had observation values with Cook's distances $>1$. between LR and SR for all years $(P<0.0001$ and $0.96<$ $R^{2}<0.99$; Fig. 2a). The linear relationships between LR and RLR $\left(P<0.05\right.$ and $0.77<R^{2}<0.82$; Fig. $\left.2 \mathrm{~b}\right)$ and SR and RLR $\left(P<0.05,0.67<R^{2}<0.81\right.$; Fig. 2c) are also significant, but weaker. Cook's distances $>1$ were identified for a small number of observation values (mainly for sites TA and FI; see Fig. 2). Removing these values from the data set did not lead to major changes in the significance of the best-fitted linear models, but their slope and $R^{2}$ values occasionally changed significantly.

\section{Simulations}

For the 500 simulations performed, we observed that the correlation between LR and SR was high $(R>0.75)$ as long as the LEP variability among patches was limited $(\sigma<0.2)$ and remained positive otherwise (Fig. 3a). The same trend was obtained when different numbers of patches were used (Appendix D: Fig. D1). We obtained strong linear relationships between LR and SR $(P<0.001)$ for three randomly chosen simulations with $0.1<\sigma<0.2$ (Fig. 3b), $0.3<\sigma<0.4$ (Fig. 3c), and $0.5<\sigma<0.6$ (Fig. 3d). The same trend was obtained when different numbers of patches were used (Appendix D: Fig. D2). As the matrices $\mathbf{M}$ were always adjusted so that the maximum eigenvalue was very close to 1 and the mean LEP value was 1 , the largest eigenvalue $\lambda_{\mathbf{C}}$ of the connectivity matrices $\mathbf{C}$ was always 1 , which was therefore also the expected slope of the LR vs. SR relationship.

Essentially identical results were obtained for the relationship between LR and RLR, with correlation coefficients decreasing as the standard deviation in larval survival rates between release sites increased.

\section{Density dependence}

When density-dependent recruitment is integrated into simulations, LR measurements based on recruits as opposed to settlers decrease at all sites as egg production (and therefore the strength of density dependence) is increased (Fig. 4a), whereas SR and RLR can either increase or decrease as a function of egg production (Fig. 4b, c). The asymptotic value of LR needed for assessing self-persistence of individual sites (Burgess et al. 2014) is only obtained when population size is small (left-hand side of Fig. 4a). The relative ordering of sites from "most" to "least" retentive can change as a function of density dependence for all three retention indices. SR and LR continue to have a proportional relationship (for each value of LEP), but the relationship between LR and RLR is perturbed, as density dependence will be perceived as changes in larval survival between release sites.

If retention indices are calculated based on counting settlers instead of recruits (not shown), then LR and RLR are unaffected by density dependence, whereas SR is affected by density dependence and values are identical to those from collecting recruits (i.e., Fig. 

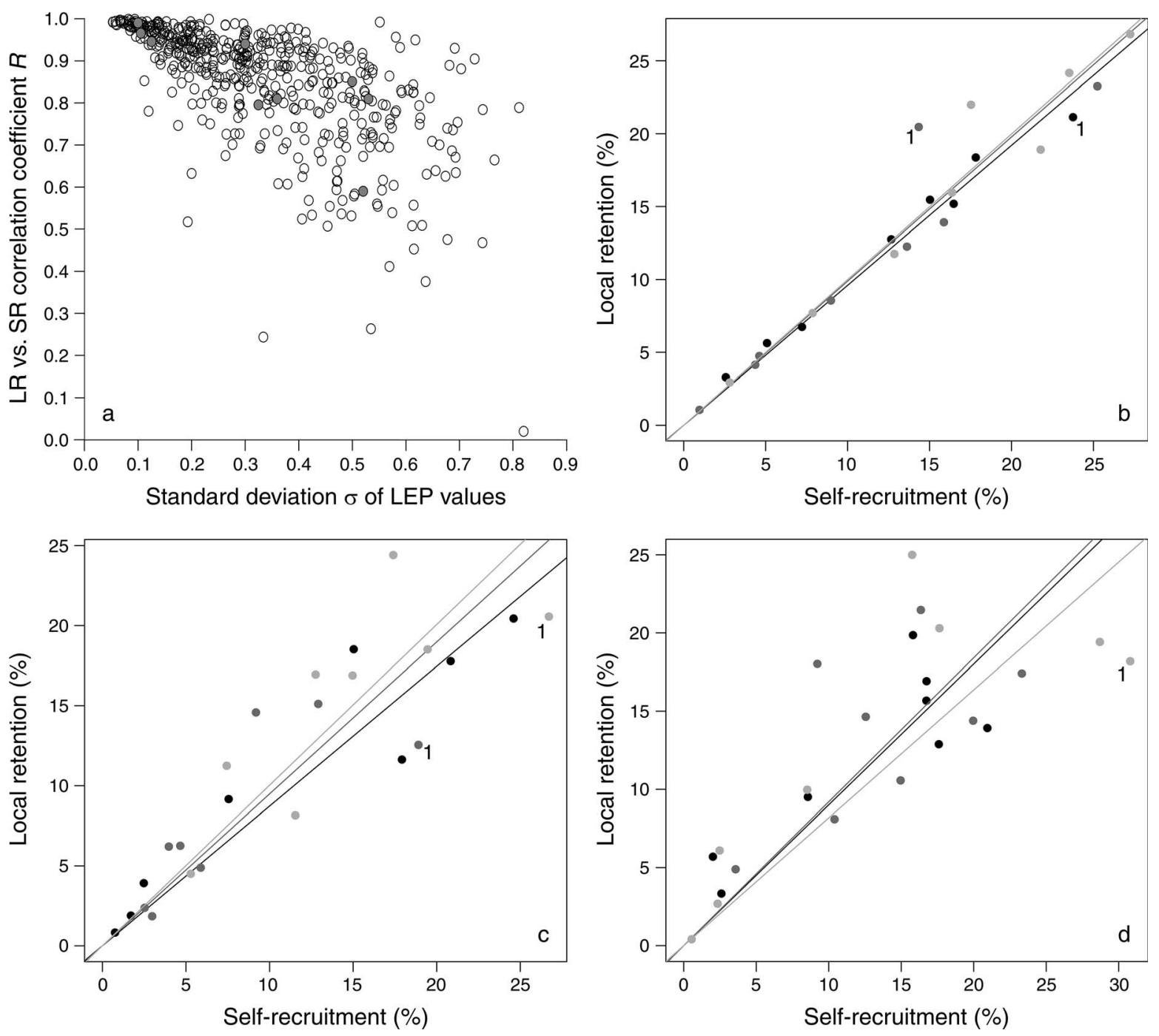

FIG. 3. Effect of spatially heterogeneous values of lifetime egg production (LEP) on the relationship between self-recruitment (SR) and local retention (LR) assessed with simulations of the dynamical system (Eq. 4). (a) Correlation coefficient $R$ of LR vs. SR asymptotic values as a function of $\sigma$, the standard deviation of LEP values among eight patches. Results from 500 simulations are shown, among which nine (solid gray circles) were randomly selected to illustrate the LR vs. SR asymptotic relationships obtained along the patches when (b) $0.1<\sigma<0.2$, (c) $0.3<\sigma<0.4$, and (d) $0.5<\sigma<0.6$. Best-fitted linear models with a slope significantly different from $0(P<0.001)$ are shown. Values with Cook's distances $>1$ are marked with 1.

4b). As a result, the proportional relationship between SR and LR is perturbed as density dependence mimics variability between sites in LEP (see Simulations and Fig. 3), whereas the relationship between LR and RLR remains unchanged.

\section{Discussion}

The potential utility of connectivity information for understanding population dynamics and persistence of reserve networks is one of the major factors driving empirical and model-based estimations of larval dispersal in marine systems (Botsford et al. 2009, Cowen and Sponaugle 2009). It has been argued that selfrecruitment (SR) does not provide useful information for assessing population persistence, but rather that local retention (LR), in combination with an estimate of lifetime egg production (LEP), is required to assess selfpersistence of individual sites and that the full connectivity matrix is needed for assessing network persistence (Burgess et al. 2014). While overall we agree with this appraisal, here we demonstrate that SR and relative local retention (RLR), which are much easier to measure in the field than LR, often provide valuable complementary information for understanding retention and connectivity in marine systems. For systems that are reasonably close to being at a stable age distribution or equilibrium state and to having LEP uniform over space, theoretical and simulation results demonstrate that there is a strong, proportional relationship between 
SR and LR (Fig. 3). The empirical comparison also indicates that there is a relationship between SR and LR (Fig. 2a), suggesting that these two conditions are close to holding for that particular system. Similarly, for systems where larval mortality rates are a relatively uniform function of release site, RLR and LR will also be proportional. Although more empirical verification is needed to test the general applicability of these results to multiple, disparate marine systems, proportional relationships between LR, SR, and RLR have a direct practical consequence: measuring SR and RLR at several sites may provide information about the relative strength of LR over sites. Though these relative rates of LR cannot, by themselves, be used to determine selfpersistence, knowing that one site is, for example, twice as retentive as another may be valuable information for implementing strategies that could maximize persistence in data-limited situations. For example, in cases where coherent ocean transport patterns generate well-defined regions over which retention rates are expected to be similar (e.g., in upwelling systems where offshore transport varies predictably, reaching a maximum near headlands and decreasing in embayments; Chavez and Messie 2009), having information about the relative strength of LR could lead to better management plans.

These results suggest a hierarchy of desirable information for assessing persistence and designing reserve networks in marine systems. Ideally, one would measure both LEP and the entire connectivity matrix to assess persistence for any arbitrary change to the pattern of LEP or connectivity. Though larval transport models can often estimate the entire connectivity matrix, these models generally use unrealistic simplifying assumptions for larval behavior and recruitment that may have important consequences for the accuracy of results (Leis et al. 2011). Unfortunately, it is rarely possible to measure the full connectivity matrix experimentally. Given these limitations, measuring LEP and LR allows one to assess self-persistence of individual sites at potentially lower experimental cost.

Nevertheless, there are numerous situations where these measurements are either not possible or will not determine persistence. As an example of the latter, consider cases where mean larval dispersal distances exceed the spatial scale of individual study sites. In these cases, no study site may be found to be self-persistent. Furthermore, if density dependence is important and only recruits can be captured, then LR measurements will not be equivalent to the LR values that determine persistence at small population size (though LR measurements with negative density dependence will be lower, and therefore provide conservative estimates of the tendency for self-persistence; see Fig. 4a). Under these circumstances, there may be value in estimating SR or RLR at multiple sites. In addition, SR measures the realized accumulation kernel into a given site (sensu Aiken et al. 2007, Shima and Swearer 2010) and RLR is a measure of the realized dispersal kernel from a given
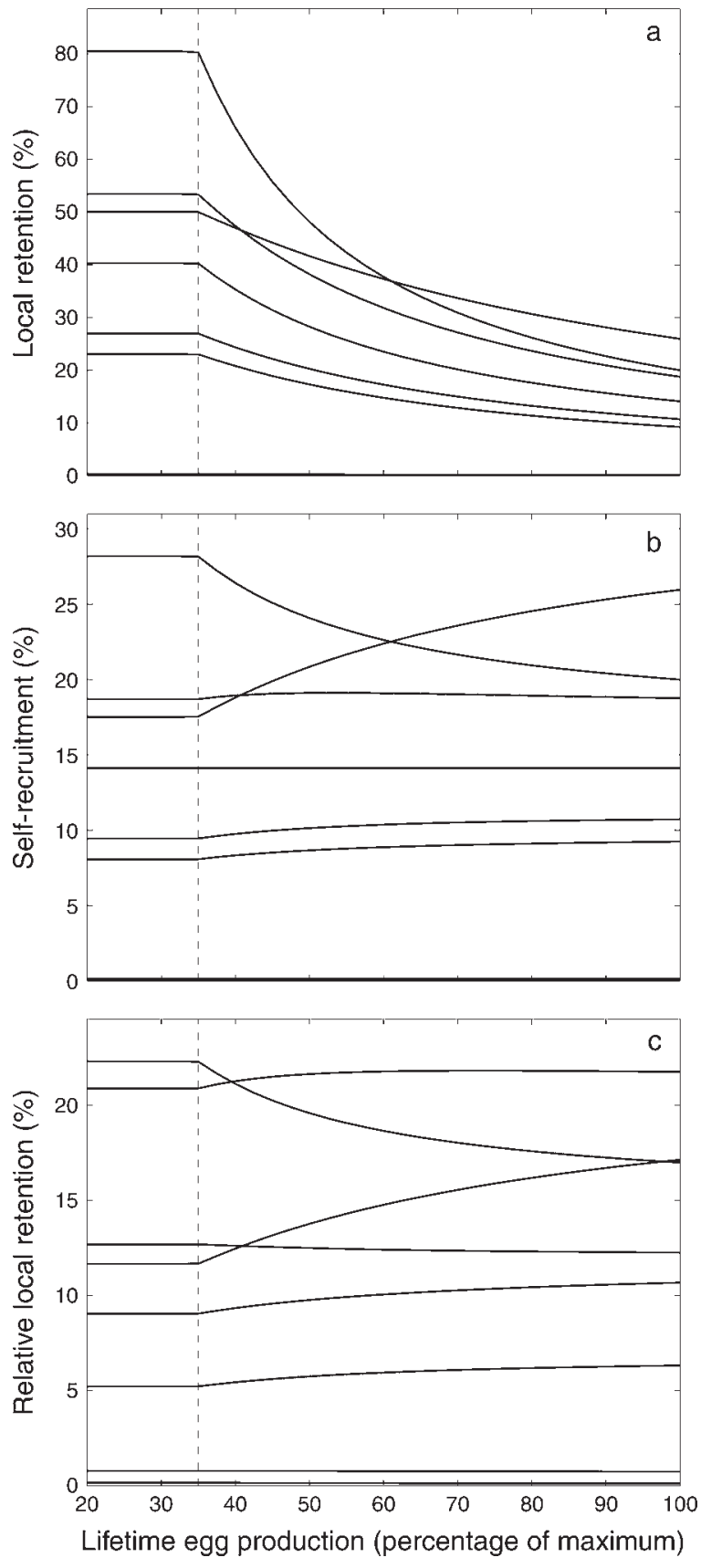

FIG. 4. (a) Local retention (LR), (b) self-recruitment (SR), and (c) relative local retention (RLR) as a function of lifetime egg production (LEP) for a randomly selected simulation with density-dependent recruitment and a random dispersal matrix. Each solid curve represents results for one of eight total patches. The vertical dashed line indicates the LEP value below which the system collapses, above which negative density dependence becomes significant.

site, so that source sites (sensu White 2008) have $\mathrm{SR}_{i}>$ $\mathrm{RLR}_{i}$ (more larvae disperse from $i$ than to $i$ ), whereas sinks have $\mathrm{SR}_{i}<\mathrm{RLR}_{i}$. Note that RLR includes recruitment to all possible habitat patches, whereas empirical studies necessarily include recruitment to a 


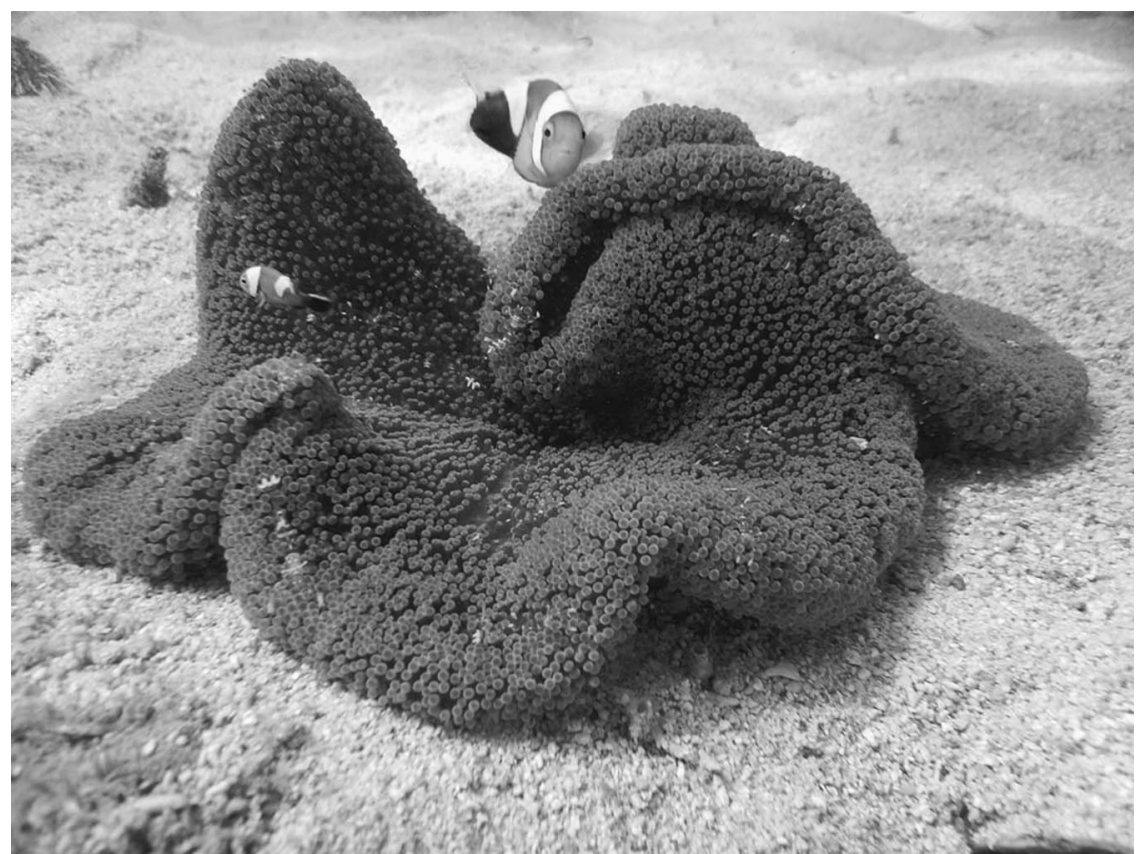

Plate 1. A pair of juvenile saddleback clownfish (Amphiprion polymnus) at their host anemone (Stichodactyla hadonni) in Bootless Bay, Papua New Guinea. Photo credit: P. Saenz-Agudelo.

limited number of sites, which could result in overestimating RLR. Consisting solely of an estimate of the fraction of larvae at a site of local origin, SR is likely the easiest of the three quantities to determine experimentally, and, therefore, may be of interest if management resources for assessing persistence are limited.

Perhaps most importantly, measuring both SR and LR provides valuable information with respect to local self-persistence and global persistence. As SR and LR are asymptotically related to each other by the dominant eigenvalue of the connectivity matrix, the ratio of these two, multiplied by LEP, can be used to test for persistence of the entire system. This global persistence test, $\mathrm{LEP} \times \mathrm{LR} / \mathrm{SR} \geq 1$, is the natural extension of that for self-persistence highlighted by Burgess et al. (2014), namely LEP $\times$ LR $\geq 1$, with SR taking into account larval input from external sources. As LR/SR is just the total recruitment divided by total egg production, this global persistence condition is just a reframing of the replacement concept, i.e., that eggs per recruit (LEP) multiplied by recruits per egg (LR/SR) must be sufficient to ensure individual replacement. It is important to note that one could just measure LEP, total recruitment, and egg production to test for global persistence, without going through the more difficult calculation of LR and SR. However, doing so does not permit assessing selfpersistence, nor would it contribute to understanding connectivity and source-sink dynamics.

Reversing this global persistence relationship allows one to estimate a critical LEP value that assures persistence of the entire population, often a highly prized piece of information for fisheries management
(Myers et al. 1999). Interestingly, this estimate does not require that all sites be visited. In practice, estimates from multiple (though not all) sites could be combined to address spatiotemporal variability in LEP and connectivity rates. As an example, 1/LR values for Saenz-Agudelo et al.'s $(2011,2012)$ data range from 2.37 to 29 , whereas the mean SR/LR value is 0.76 , indicating that between three and 38 times less LEP is necessary to assure global persistence than self-persistence of individual sites. This highlights the important contribution of larval exchange between sites for persistence in this system.

It is important to note that these results depend on LEP being relatively uniform over space. Ideally, empirical connectivity studies should estimate LEP at multiple study sites, though this may not always be feasible. Factors contributing to spatially inhomogeneous LEP values include heterogeneous exploitation effort, e.g., due to the presence of marine protected areas (MPAs). Therefore, this type of analysis is more appropriate for systems for which MPAs have not yet been established. Simulations are a useful approach to assessing the effects of spatially inhomogeneous LEP values on SR vs. LR correlation levels (Fig. 3a; Appendix D: Fig. D1), and ultimately on the usability of our condition of global metapopulation persistence in the field.

Ultimately, the decision of how best to assess connectivity in a given system depends on numerous factors, including the intended use of this information (e.g., population dynamics or genetic connectivity), available resources, and the biological details of the system. This study provides a conceptual framework for 
assessing some of the benefits and limitations of different connectivity indices, and maximizing the value of information collected. It is our hope that this framework forms the basis for future decisions regarding investment of scientific resources for measuring connectivity and dispersal in marine systems.

\section{ACKNOWLEDGMENTS}

This work was supported by the COMPO project (Connectivity Of Marine POpulations, http://www.compo.ird.fr) through a grant from the French National Research Agency (ANR), grant number 2010 JCJC 170101 . We thank J. Wilson White and an anonymous reviewer for their useful comments.

\section{Literature Cited}

Aiken, C. M., S. A. Navarrete, M. I. Castillo, and J. C. Castilla 2007. Along-shore larval dispersal kernels in a numerical ocean model of the central Chilean coast. Marine Ecology Progress Series 339:13-24.

Almany, G. R., M. L. Berumen, S. R. Thorrold, S. Planes, and G. P. Jones. 2007. Local replenishment of coral reef fish populations in a marine reserve. Science 316:742-744.

Botsford, L. W., J. W. White, M. A. Coffroth, C. B. Paris, S. Planes, T. L. Shearer, S. R. Thorrold, and G. P. Jones. 2009. Connectivity and resilience of coral reef metapopulations in marine protected areas: matching empirical efforts to predictive needs. Coral Reefs 28:327-337.

Burgess, S. C., K. J. Nickols, C. D. Griesemer, L. A. K. Barnett, A. G. Dedrick, E. V. Satterthwaite, L. Yamane, S. G. Morgan, J. W. White, and L. W. Botsford. 2014. Beyond connectivity: how empirical methods can quantify population persistence to improve marine protected area design. Ecological Applications 24:257-270.

Carson, H. S., P. C. López-Duarte, L. Rasmussen, D. Wang, and L. A. Levin. 2010. Reproductive timing alters population connectivity in marine metapopulations. Current Biology 20: 1926-1931.

Chavez, F. P., and M. Messié. 2009. A comparison of Eastern Boundary Upwelling Ecosystems. Progress in Oceanography 83:80-96.

Cowen, R. K., K. M. M. Lwiza, S. Sponaugle, C. B. Paris, and D. B. Olson. 2000. Connectivity of marine populations: open or closed? Science 287:857-859.

Cowen, R. K., and S. Sponaugle. 2009. Larval dispersal and marine population connectivity. Annual Review of Marine Science 1:443-466.

Cuif, M., D. M. Kaplan, J. Lefèvre, V. M. Faure, M. Caillaud, P. Verley, L. Vigliola, and C. Lett. 2014. Wind-induced variability in larval retention in a coral reef system: a biophysical modelling study in the South-West Lagoon of New Caledonia. Progress in Oceanography 122:105-115.

Hamilton, S. L., J. Regetz, and R. R. Warner. 2008. Postsettlement survival linked to larval life in a marine fish. Proceedings of the National Academy of Sciences USA 105: $1561-1566$.

Hixon, M. A., S. W. Pacala, and S. A. Sandin. 2002. Population regulation: historical context and contemporary challenges of open vs. closed systems. Ecology 83:1490-1508.
Hogan, J. D., R. J. Thiessen, P. F. Sale, and D. D. Heath. 2012. Local retention, dispersal and fluctuating connectivity among populations of a coral reef fish. Oecologia 168:61-71.

Jones, G. P., M. J. Milicich, M. J. Emslie, and C. Lunow. 1999. Self-recruitment in a coral reef fish population. Nature 402: 802-804.

Jones, G. P., S. Planes, and S. R. Thorrold. 2005. Coral reef fish larvae settle close to home. Current Biology 15:1314-1318.

Kaplan, D. M., L. W. Botsford, and S. Jorgensen. 2006. Dispersal per recruit: an efficient method for assessing sustainability in marine reserve networks. Ecological Applications 16:2248-2263.

Kaplan, D. M., L. W. Botsford, M. R. O'Farrell, S. D. Gaines, and S. Jorgensen. 2009. Model-based assessment of persistence in proposed marine protected area designs. Ecological Applications 19:433-448.

Leis, J. M., L. Van Herwerden, and H. M. Patterson. 2011. Estimating connectivity in marine fish populations: what works best? Pages 193-234 in R. N. Gibson, R. J. A. Atkinson, and J. D. M. Gordon, editors. Oceanography and marine biology: an annual review. Volume 49. CRC Press, Boca Raton, Florida, USA.

Myers, R. A., K. G. Bowen, and N. J. Barrowman. 1999. Maximum reproductive rate of fish at low population sizes. Canadian Journal of Fisheries and Aquatic Sciences 56: 2404-2419.

Pineda, J., J. A. Hare, and S. Sponaugle. 2007. Larval transport and dispersal in the coastal ocean and consequences for population connectivity. Oceanography 20:22-39.

Pinsky, M. L., S. R. Palumbi, S. Andréfouët, and S. J. Purkis. 2012. Open and closed seascapes: where does habitat patchiness create populations with high fractions of selfrecruitment? Ecological Applications 22:1257-1267.

Planes, S., G. P. Jones, and S. R. Thorrold. 2009. Larval dispersal connects fish populations in a network of marine protected areas. Proceedings of the National Academy of Sciences USA 106:5693-5697.

R Development Core Team. 2012. R: a language and environment for statistical computing. R Foundation for Statistical Computing, Vienna, Austria. http://www. R-project.org/

Saenz-Agudelo, P., G. P. Jones, S. R. Thorrold, and S. Planes. 2011. Connectivity dominates larval replenishment in a coastal reef fish metapopulation. Proceedings of the Royal Society B 278:2954-2961.

Saenz-Agudelo, P., G. P. Jones, S. R. Thorrold, and S. Planes. 2012. Patterns and persistence of larval retention and connectivity in a marine fish metapopulation. Molecular Ecology 21:4695-4705.

Shima, J. S., and S. E. Swearer. 2010. The legacy of dispersal: larval experience shapes persistence later in the life of a reef fish. Journal of Animal Ecology 79:1308-1314.

Vigliola, L., and M. G. Meekan. 2002. Size at hatching and planktonic growth determine post-settlement survivorship of a coral reef fish. Oecologia 131:89-93.

White, J. W. 2008. Spatially coupled larval supply of marine predators and their prey alters the predictions of metapopulation models. American Naturalist 171:E179-E194.

\section{Supplemental Material}

\section{Ecological Archives}

Appendices A-D are available online: http://dx.doi.org/10.1890/14-1305.1.sm 


\section{ERRATUM}

Christophe Lett and colleagues discovered two mistakes that appeared in their paper (C. Lett, T. Nguyen-Huu, M. Cuif, P. Saenz-Agudelo, and D. M. Kaplan. 2015. Linking local retention, self-recruitment, and persistence in marine metapopulations. Ecology 96:2236-2244) published in the August issue. In the legend of Fig. 1, the 4th line should read "... at patch $i$ and $c_{j i}$ is the proportion ..." instead of "... at patch $i_{j}$ and $c_{i j}$ is the proportion ...." These errors were introduced in the editorial process and we apologize for any inconvenience this may have caused. 